\title{
Representational Use on a Lab Question by Modeling Workshop Participants
}

\author{
Kathleen A. Harper, ${ }^{1}$ Ted M. Clark, ${ }^{2}$ Lin Ding, ${ }^{3}$ and Matthew A. Kennedy ${ }^{4}$ \\ ${ }^{1}$ Department of Engineering Education, The Ohio State University, 2070 Neil Ave, Columbus, OH 43210 \\ ${ }^{2}$ Department of Chemistry and Biochemistry, The Ohio State University, $100 \mathrm{~W} .18^{\text {th }}$ Ave, Columbus, OH \\ $43210{ }^{3}$ Department of Teaching and Learning, The Ohio State University, 1945 N. High St, Columbus, OH \\ $43210{ }^{4}$ Laurel School, One Lyman Circle, Shaker Heights, OH 44122
}

\begin{abstract}
Like many research-informed science curricula, Modeling Instruction (MI) emphasizes using multiple representations to learn science and solve problems. Also like many reformed curricular approaches, MI evaluations have primarily focused on conceptual gains. This is particularly true when investigating the effect of teacher professional development based upon MI. While such efforts are valuable, there is a need to broaden the assessment of professional development offerings. To address this need, Modeling workshop participants were given "lab questions" from a recent AP physics exam. These responses were scored by a trained AP reader and analyzed qualitatively. The results show that the participants not only scored better than the national average, but that the participants tended to use representations appropriate to the questions posed.
\end{abstract}

\section{BACKGROUND AND CONTEXT}

Modeling Instruction [1, 2] has been the backbone of a class of science teacher professional development workshops for approximately 25 years. What began as a collaboration between a high school teacher and physics professor in Phoenix, AZ has grown to reach thousands of teachers throughout the country and world. Detailed accounts of the Modeling approach can be found elsewhere [2,3], but briefly, the content is organized around core scientific models. Students develop these models through carefully guided inquiry. Each topic begins with observations. Then students design an experiment to explore relationships between measurable quantities. The teacher insures they arrive at experiments that will generate quality data, which students represent and analyze to obtain graphical and mathematical relationships between the quantities of interest. The models are then applied to solve problems and make predictions. Throughout, students are encouraged to engage in scientific discourse. Two Modeling elements that are core to this study are:

(A) the empirical basis of generating scientific knowledge, and

(B) the central role of multiple representations in scientific analysis and problem solving.

The workshop structure alternates between "student mode" and "teacher mode." In student mode, participants are led through activities in the Modeling framework by leaders who are experienced K-12
Modeling teachers. Participants try to imitate student reasoning and learning. In teacher mode, participants debrief on the experience, ask the leaders about the rationale for certain aspects of the approach, and discuss how they might make adjustments for their own environments.

Modeling workshops in physics, chemistry, and Modeling curriculum development have been offered through The Ohio State University since 2004 [4]. In the first 13 years of the program over 400 different teachers participated, with approximately $1 / 3$ of those teachers taking multiple workshops. Assessment has been extremely positive. Qualitative responses on the external evaluator's surveys show that every participant would recommend the workshop to a fellow teacher and that all but one stated they learned new content and/or skills. These surveys also indicate many areas of pedagogical growth for the teachers. In quantitative analysis, average normalized gains on the Force Concept Inventory (FCI) [5] were 0.45 . ( $\mathrm{N}=$ 224) These results make it clear that participants have gained significant conceptual understanding through the workshops and also feel they have benefitted pedagogically. However, these assessment methods do not specifically address the two core elements of Modeling identified above.

This work looked to address the following:

(1) Could an assessment of experimental design and analysis be developed that could be administered relatively easily?

(2) If such an assessment were given to Modeling 
workshop participants, would they exhibit the behaviors the workshop sought to emphasize?

\section{THE ASSESSMENT AND ITS ADMINISTRATION}

In recent years, the Advanced Placement (AP) examinations in science have included questions that focus on experimental design and data analysis. For the purposes of this work, a question that appeared on the 2016 U. S. Main form of the Physics 1 exam looked promising. This problem is summarized in Fig. 1 and can be found in full detail on the AP Central website.

Part A) Design an experiment to test whether a toy ball bounces perfectly elastically at low speeds, but not at high speeds

Part B) Describe how to represent the data in a graph or table and how to use it to evaluate the hypothesis.

Part C) If the collision were elastic at low speeds, but violated a law of physics at high speeds, what would the representation look like?

FIG 1. Summary of lab question from 2016 U. S. Main form of the AP Physics 1 exam [6].

Note that part A ties to point A) made above about the Modeling approach, the role of experimental design in science, and that part B relates to point B), the use of representations.

This problem was given to the participants on the last day of the 2016 physics workshop, without informing them that it was a question from an AP exam. $(\mathrm{N}=15)$ Immediately after the responses were collected, the participants were asked to respond to the following prompt: "independent of how you feel you performed on the previous question, were there any elements of the modeling instruction curriculum that influenced your approach to solving the question? If so, how? If not, why?" The answers to the laboratory question were scored by an AP reader who had graded hundreds of student responses to this question for the College Board [7]. Additionally, three researchers read through these responses, as well as the reflections, to identify trends and develop a qualitative coding scheme that was applied to the data.

The workshop participants taught in a wide variety of settings, spanning from urban to rural, lowincome to affluent, and included charters, private, and public schools. Further, their experience varied, ranging from 0 to 24 years of classroom experience, with an average number of years in the classroom of 7.6. One had just finished her student teaching, and another was a gifted intervention specialist for her district with a science background. As is often the case, many people in the group taught a variety of science courses. All 13 participants that had been in the classroom the preceding year had taught physics and/or physical science. They also taught biology, chemistry, and a variety of other courses. One participant taught exclusively conceptual physics. Only two participants had taught AP physics; the others did not appear to have seen the assessment question before, though they were not specifically asked if they had or not.

\section{ASSESSMENT RESULTS AND FINDINGS}

The average normalized gain on the FCI for the entire group over the workshop was 0.31 , including 12 post-test scores of 25 or higher, indicating the workshop impacted their understanding of Newtonian mechanics.

The distribution of scores on the AP question is shown in Fig. 2. The mean is 6.9 out of 12, and the median is 7 , with scores spanning the entire range (from 0 to 12). The average score attained nationally by AP students on this exam was 4.3 .

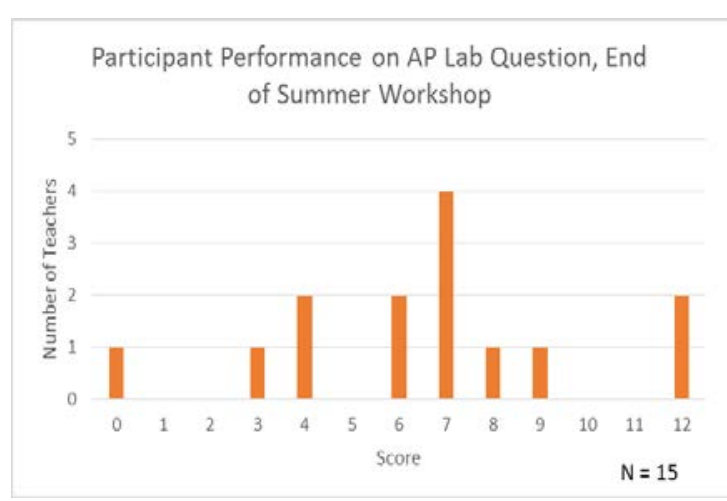

FIG 2. Scores of workshop participants on assessment at the end of the workshop. 
The participants showed a strong ability to design and describe a valid experiment, averaging 3.4 out of a possible 4 points on part A. All but one participant chose an approach that would generate the appropriate data to answer the question posed. They also tended to employ useful representations, scoring an average of 2.6 out of 4 on part B. Part C only averaged 0.9 of 4 . Performance on the assessment was unrelated to years of teaching experience, type of courses taught (including whether or not the teacher had taught AP), type of school taught at, or FCI score.

The representations generated in part $\mathrm{B}$ are summarized in Table I. The numbers do not add up to 15 , as some participants used more than one type.

TABLE I. Representations employed by participants.

\begin{tabular}{cc}
\hline Representation Type & Number \\
\hline Bounce height vs. drop height & 9 \\
Another valid graph & 6 \\
Work-energy bar chart & 3 \\
\hline
\end{tabular}

The most common representation, graphing bounce height vs. drop height, leads to a straightforward analysis of the posed question, since if the ball behaved perfectly elastically, the slope of the graph would be 1 . The fact that a majority of the respondents employed such a representation is particularly noteworthy, since it is not a "standard" graph shown in any physics textbook. One participant even said in his reflection that prior to the workshop he would have thought such a graph "meaningless," but it became the focus of his response. It is also noteworthy that those who used this approach outscored those who did not, with an average score of 9 points, as opposed to 3.8 points, on the problem.

A major emphasis in MI is interpreting the slopes of graphs to describe physical situations, and nearly half of the participants (7 out of 15) specifically discussed how to use the slope of the graph they generated to answer the question.

In the participant reflections, 14 specifically said the workshop influenced their answer to the problem and 1 was unclear as to whether the answer was yes or maybe. Table II summarizes the most common aspects of the workshop cited by participants in their reflections. Again, many responses mentioned multiple characteristics of the workshop.
TABLE II. Workshop influences identified by participants.

\begin{tabular}{cc}
\hline Aspect & Number \\
\hline Graphing & 10 \\
Energy conservation & 4 \\
approach & 3 \\
Work-energy bar charts & 3 \\
Visual representation use & \\
\hline
\end{tabular}

Other aspects of the workshop mentioned by participants included using slopes, using appropriate mathematical relationships, thinking conceptually or qualitatively in approaching problems, thinking about how the data should look, and remembering the details of one particular lab from the workshop.

A theme connecting the majority of these responses is representations, supporting the idea that the workshop effectively illustrated the use of representations to analyze scientific situations. As one participant wrote, "Modeling has influenced me to use visual representations of the data to show the relationship[s], so I used a graph to show how bounce height was related to the speed." There is also some evidence that participants found the workshop's emphasis on experimental process (both design and analysis) helpful in approaching this task. For instance, one reflection from a participant who is also a very experienced physics teacher stated that "Modeling made me question how I conducted the experiment in much more detail.”

\section{DISCUSSION}

This study suggests that AP physics lab questions are well suited for assessing science teachers' approaches for solving problems involving experimental design and analysis. Even though such questions are obviously intended for assessing AP students, this investigation shows they can provide useful information about other populations, like participants in a professional development workshop. As described above, participant's FCI scores were much higher than those of typical high school students, and this study included several veteran teachers. Also, these individuals had been immersed in an intense 3-week workshop with a track record of success, taught by experienced leaders. However, even for this population in this setting, the scores spanned a wide range and a ceiling effect was not evident.

In terms of the central role of multiple representations 
in scientific analysis and problem solving in Modeling workshops, participants both used such representations in their own problem solving and, in their reflections, valued representations like graphs and bar charts. Moreover, the use of one particular graphical representation (bounce height vs. drop height) was associated with greater success on the overall assessment. Analysis of participants' use of multiple representations and their associated reflections indicate that the workshop did indeed influence these teachers to use new representational tools for laboratory analysis. Further, a few participants specifically stated that thinking about the representations they would use guided the design of their experiments. As multiple representations and experimental design are central features of Modeling workshops, it is encouraging to see them mentioned together in this context.

There are some limitations on the conclusions that can be drawn from this study. First, this is one small sample of teachers. This was intentional in that all of the participants were in the same cohort and received the same instruction during the workshop, but it does lead to a small sample size. Second, one of the activities that the instructors of the workshop used to introduce scientific ways of thinking was an exercise that involved bouncing balls of various elasticities and making some graphs, though not necessarily the type of graph that was employed by the teachers on this assessment. This is the lab that was mentioned by a couple of teachers in their reflection as something influencing their approach. For this reason, it is not clear the extent to which participants are transferring skills involving the use of representations for problem solving. Third, since this question was only administered at the end of the workshop, it is not clear if participants changed or improved their approach as a result of the workshop.
In other words, some of the participants may have utilized multiple representations when answering this question before the workshop. Finally, this question was administered while the participants were still at the workshop and in "workshop mode," so it is unknown whether this effect was temporary or more lasting.

There are many opportunities to extend this work and address some of the uncertainties above. First, administering a second similar question to these teachers during the school year would help address the question of whether the influence of the workshop fades or not. Additionally, the similarity of the question context to an activity from the workshop should be probed; two possibilities include asking a question in a less familiar context or changing the introductory activity in a future version of the workshop.

Another question is how the responses of workshop participants would compare to those of people who had not taken the workshop; this could be addressed by administering the question in a preand post- format in a future year or by giving it to teachers who have not been to the workshop.

Finally, a big unanswered question is whether the students of these teachers would exhibit similar approaches on an assessment like this. Several former participants have agreed to administer the question to their students and share the responses with the research team.

\section{ACKNOWLEDGEMENTS}

The authors thank the staff of the Central Ohio Science Modeling Workshops, particularly physics workshop leaders Brian Carpenter and Chas Deremer, as well as the workshop participants.
[1] M. Wells, D. Hestenes \& G. Swackhamer, Am. J. Phys. 63 (1995).

[2] D. Hestenes, Modeling methodology for physics teachers, in E. Redish \& J. Rigden (Eds.) The Changing Role of the Physics Department in Modern Universities (American Institute of Physics, 1997).

[3] D. Hestenes, Am. J. Phys. 55 (1987).
[4] K.A. Harper \& T.M. Clark, American Modeling Teachers Association spring 2017 newsletter (AMTA, 2017); J.M. Cervenec \& K.A. Harper, in Proc. Phys. Ed. Res. Conf. 2005 (AIP, 2006).

[5] D. Hestenes, M. Wells \& G. Swackhamer, Phys. Teach., 30 (1992).

[6] The College Board, AP Physics 1: Algebra-Based 2016 Free-Response Questions. Retrieved from www.collegeboard.org

[7] The process of training an AP reader is rigorous, leading to high reliability. 